Tн е ability to attribute mental states to others ('theory of mind') pervades normal social interaction and is impaired in autistic individuals. In a previous positron emission tomography scan study of normal volunteers, performing a 'theory of mind' task was associated with activity in left medial prefrontal cortex. We used the same paradigm in five patients with Asperger syndrome, a mild variant of autism with normal intellectual functioning. No task-related activity was found in this region, but normal activity was observed in immediately adjacent areas. This result suggests that a highly circumscribed region of left medial prefrontal cortex is a crucial component of the brain system that underlies the normal understanding of other minds.

Key words: Autism; Brain imaging; Brodmann area $8 / 9$ Frontal cortex; Theory of mind

\section{Theory of mind' in the brain. Evidence from a PET scan study of Asperger syndrome}

\author{
Francesca Happé, 1,2 Stefan Ehlers, ${ }^{3}$ \\ Paul Fletcher, ${ }^{4}$ Uta Frith, ${ }^{1,2}$ \\ Maria J ohansson, ${ }^{3}$ \\ Christopher Gillberg, ${ }^{3}$ Ray Dolan, ${ }^{4}$ \\ Richard Frackowiak ${ }^{4}$ \\ and Chris Frith ${ }^{2,4, C A}$
}

${ }^{1}$ MRC Cognitive Development Unit, 4 Taviton Street, London WC1 OBT; ${ }^{2}$ Psychology

Department, University College London, Gower Street, London WC1E 6BT; ${ }^{3}$ Department of Child and Adolescent Psychiatry, University of Goteborg, Sweden; ${ }^{4}$ Wellcome Department of Cognitive Neurology, Institute of Neurology, 12 Queen Square, London WCIN 3BG, UK

${ }^{C A}, 4$ Corresponding $A$ uthor and $A$ ddress

\section{Introduction}

Theory of mind, a somewhat misleading catch phrase, is used to refer to our everyday ability to attribute mental states to ourselves and others in order to predict and explain behaviour. ${ }^{1}$ This ability has become a focus of interest for cognitive scientists, primatologists and philosophers as well as for clinicians dealing with a puzzling mental handicap: autism. ${ }^{2}$ Individuals with autism appear to lack the basic ability to construe behaviour in terms of mental states (mentalizing), and have marked difficulty with simple tasks requiring attribution of, for example, a false belief to a story character. ${ }^{3,4}$ There is evidence that autism is associated with abnormal brain development, ${ }^{5-7}$ and this has prompted the idea that there is an innate brain-based mechanism which underlies the normal development of mentalizing. ${ }^{8}$ In addition, since some individuals with autism do well on many cognitive tasks, but still have problems in mentalizing, the brain system that is critically involved in the development of mentalizing may be relatively discreet and vulnerable to selective damage. ${ }^{9}$

In a previous position emission tomography (PET) scan study with normal volunteers ${ }^{10}$ we compared the pattern of brain activity while reading stories involving complex mental states with the pattern of activation in two control tasks: reading non-mental stories and unconnected sentences. U sing a subtraction technique, where we compare all three conditions, the brain region most specifically associated with mentalizing story comprehension, over and above more general story comprehension, was in the left medial prefrontal cortex, on the border between Brodmann areas 8 and 9 . The same region has been identified by another group using a very different story comprehension mentalizing task. ${ }^{11}$

Clearly it would be informative to scan people with autism using the same task. It would be meaningless, however, to scan subjects attempting a task they cannot perform; most individuals with autism cannot think about thoughts and feelings in the way these story tasks require. ${ }^{12}$ A small minority, however, do pass standard tests of false belief attribution. Some of these individuals, who tend to be older and more verbal than other autistic subjects ${ }^{13}$ show remarkable success across an array of tests that involve mentalizing. ${ }^{14}$ These individuals often receive the diagnosis of A sperger syndrome. ${ }^{15}$ Their continuing social difficulties in everyday life and their 
tell-tale slips in more naturalistic mentalizing tests $^{16}$ might reflect delay in development of this cognitive component, and suggest that different processes underlie mental state attribution in these individuals.

\section{Subjects and Methods}

Subjects were five right-handed males, mean age 24 (range 20-27) years. All had been diagnosed with A sperger syndrome on the basis of developmental history and current presentation (marked social abnormality, good language in the presence of verbal and non-verbal communication difficulties, presence of circumscribed special interests, resistance to change). A history of clumsiness was present in four of the patients, but otherwise clinical examination revealed no neurological abnormalities. The mean WAIS Full Scale IQ for the group was 100 (range 87-112), the mean VIQ was 110 (93-125), mean PIQ

Table 1 Tasks used during scanning and rationale of subtraction design

\begin{tabular}{lllll}
\hline Condition & Reading & Memory & $\begin{array}{l}\text { Integration } \\
\text { and } \\
\text { inference }\end{array}$ & $\begin{array}{l}\text { Mental } \\
\text { state } \\
\text { attribution }\end{array}$ \\
\hline ToM & + & + & + & + \\
Phys & + & + & + & - \\
US & + & + & - & - \\
\hline
\end{tabular}

Example Mentalizing (ToM) story: A burglar who has just robbed a shop is making his getaway. As he is running home, a policeman on his beat sees him drop his glove. He doesn't know the man is a burglar, he just wants to tell him he dropped his glove. But when the policeman shouts out to the burglar, "Hey, you! Stop!", the burglar turns round, sees the policeman, and gives himself up. He puts his hands up and admits that he did the break-in at the local shop.

Q: Why did the burglar do this?

Example Physical (Phys) story: A burglar is about to break into a jewellers' shop. He skillfully picks the lock on the shop door. Carefully he crawls under the electronic detector beam. If he breaks this beam it will set off the alarm. Quietly he opens the door of the store-room and sees the gems glittering. As he reaches out, however, he steps on something soft. He hears a screech and something small and furry runs out past him towards the shop door. Immediately the alarm sounds.

\section{Q: Why did the alarm go off?}

Example Unlinked sentences (US): The four brothers stood aside to make room for their sister, Stella. Gill repeated the experiment, several times. The name of the airport has changed. Louise uncorked a little bottle of oil. The two children had to abandon their daily walk. She took a suite in a grand hotel. It was already twenty years since the operation.

Q: Did the children take their walk?
92 (83-100). Magnetic resonance imaging scans showed no evidence of gross morphological abnormalities, and all volunteers were free from epilepsy and were not currently taking any medication. The study was approved by the local hospital ethics committee, and permission to administer radioactive substances was obtained from the Advisory Committee on Radioactive Substances (ARSAC) UK.

We used exactly the same story comprehension paradigm as previously used with normal volunteers. ${ }^{10}$ Thus there were physical stories (Phys), stories requiring mentalizing (TOM), and unconnected sentences (US). Examples of the stories are provided in table 1 . The task design aimed at separating out the task demands for story integration ((ToM + Phys) US) and mental state attribution (ToM - Phys). The three types of text were presented on a computer monitor. Each of the three conditions was administered four times over the 12 scans in an $A B C$ counterbalanced design. D uring each scan two stories were presented. Subjects were given practice passages before beginning, and were told before each scan which type of passage they would be shown. Subjects were required to read the passage silently and, when ready, to touch the screen. At this point the passage was removed and a question appeared which was to be answered silently.

Since the stories were Swedish, Swedish translations of the story materials were used, validated with 10 normal Swedish subjects. This validation showed results very similar to data from 60 English subjects tested on the original English versions. Scores for the three conditions were $13.5,12.8$, and 12.4 of a maximum of 16, compared with 12.9, 12.8, 12.2 in the English sample. We ther efore conclude that the materials were comparable across the languages, as well as being comparable across conditions.

Scans were obtained using a CTI model 953B PET scanner (CTI, K noxville, U SA) with colimating septa retracted (FWHM $8 \mathrm{~mm}$ transaxial, $4 \mathrm{~mm}$ axial). Volunteers received a $20 \mathrm{~s}$ bolus of $\mathrm{H}_{2}{ }^{15} \mathrm{O}(55 \mathrm{M} \mathrm{Bq}$

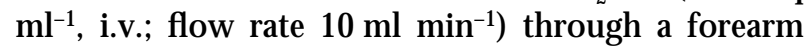
cannula. The data were analysed with statistical parametric mapping (SPM 95 software from the W ellcome D epartment of Cognitive $\mathrm{N}$ eurology, London) implemented in $M$ atlab ( $M$ athworks, Sherborne, MA). The scans from each subject were realigned using the first as a reference. A $s$ a final preprocessing step the image were smoothed using an isotropic $G$ aussian kernel. The condition, subject and covariate effects (global blood flow) were estimated according to the general linear model at each voxel. To test hypotheses about regionally specific condition effects, the estimates were compared using linear compounds or contrasts. ${ }^{17}$ 

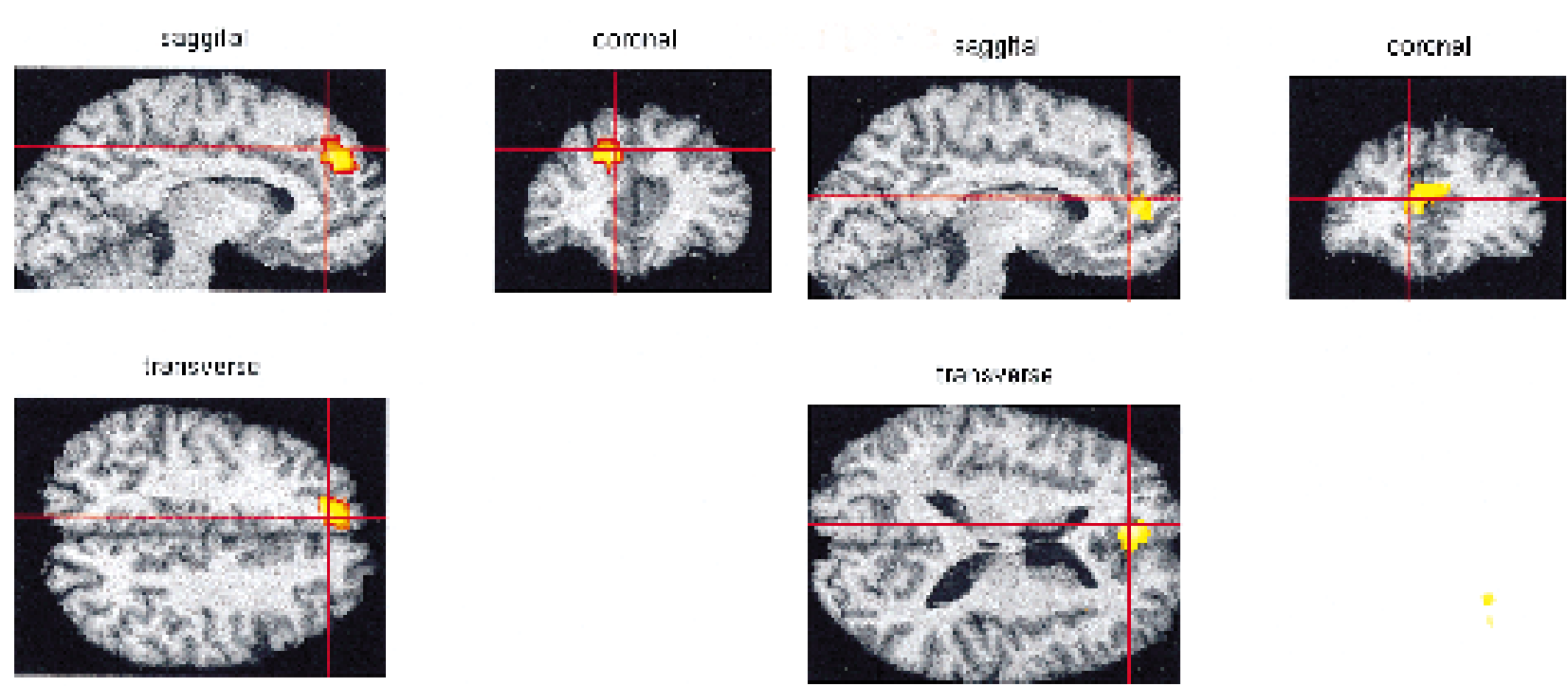

FIG. 1. The location of the peak activation in medial prefrontal cortex when theory of mind stories were compared with physical stories Regions of significantly increased activity as measured by PET are shown superimposed on a standard MRI image that had been normalized into the stereotactic space of Tallairach \& Tournoux. Saggital, coronal and horizontal views are shown. The left-hand panel shows the result for six normal volunteers. There was a significant activation on the border between Brodmann areas 8 and 9 (coordinates -12 , 36, 36; $Z=4.1$ ). The right-hand panel shows the results for five volunteers with Asperger syndrome. There was no increase in activity while reading ToM stories in the area of peak activity in the normal volunteers and this difference between groups was significant. There was, however, an increase in a more inferior region of medial prefrontal cortex (coordinates; $-10,44,16 ; Z=2.38$ ).

Table 2. Performance on tasks during scanning

\begin{tabular}{lccrr}
\hline Condition & Time $^{(\mathrm{s})^{\mathrm{a}}}$ & Range & Score $^{\mathrm{b}}$ & Range \\
\hline Asperger & & & & \\
ToM & $42.2 \pm 16.4$ & $28-67$ & $11.4 \pm 3.4$ & $8-15$ \\
Phys & $51.6 \pm 23.8$ & $28-85$ & $11.0 \pm 3.7$ & $5-14$ \\
US & $37.7 \pm 11.9$ & $25-51$ & $12.4 \pm 3.2$ & $8-14$ \\
Normal & & & & \\
ToM & $33.7 \pm 6.2$ & $21-49$ & $15.4 \pm 0.4$ & $15-16$ \\
Phys & $35.0 \pm 5.8$ & $23-44$ & $14.2 \pm 0.8$ & $13-15$ \\
US & $32.2 \pm 4.1$ & $23-39$ & $9.6 \pm 3.2$ & $6-14$ \\
\hline
\end{tabular}

aHarmonic mean \pm s.d.

bMean \pm s.d.; maximum possible $=6$.

Column 1 shows the average time taken to read a passage; column 3 shows the total scores obtained for answers to questions about the passages. Scores for each answer were between 0 and 2 .

Table 3. Results of scanning: Areas of significant activation in patients with Asperger Syndrome

\begin{tabular}{lrrr}
\hline Brain region & Coordinates & $\mathrm{z}$ & $\mathrm{p}$ \\
\hline ToM vs US & & & \\
Medial prefrontal (9/10) & $2,50,20$ & 2.86 & 0.002 \\
Temporal pole (left 21) & $-52,0,-16$ & 3.09 & 0.001 \\
Temporal pole (right 21) & $44,-8,-16$ & 2.74 & 0.003 \\
Angular gyrus (left 22/39) & $-50,-56,16$ & 2.32 & 0.010 \\
& & & \\
ToM vs Phys & & & \\
Medial prefrontal (left 9/10) & $-10,44,16$ & 2.38 & 0.000 \\
\hline
\end{tabular}

\section{Results}

Table 2 shows times to read and comprehend each passage and scores for the answers to the test questions which were recorded in between scans. A score of 0 was given for answers of 'don't know' or incorrect answers, 1 for partially correct answers, and 2 for fully correct answers.

The average time taken in each condition by the individuals with A sperger syndrome was more variable but not significantly different from the time taken by the normal volunteers. Scores on ToM stories were significantly worse $(F(1,9)=10.8, p<$ 0.01). Importantly, there were no significant differences in the scores for the other conditions. The Phys stories tended to be slightly more difficult than the ToM stories, and the patient group had slightly better scores for the unlinked sentences.

$B$ rain regions activated in volunteers with $A$ sperger Syndrome comparing theory of mind task with the control tasks are shown in table 3. With one exception, the same areas were significantly activated in the A sperger subjects as in the normal volunteers. The coordinates show the position for the peak activation. $D$ ifferences in activation between conditions were significantly smaller in the Asperger group when compared directly with the control group. All activations were in the same location, how ever, taking into account the spatial resolution of PET. 
The notable exception to the above concerns the medial prefrontal cortex. The area activated in the control group (area 8: $x y z=-10,40,36$ ) was not activated in the patients with A sperger, and this difference between groups was significant $(z=2.12, p<$ 0.02 ). The A sperger patients did, however, show activation of an adjacent, but more ventral area of medial prefrontal cortex, area 9/10.

Figure 1 illustrates the pattern of activation in A sperger syndrome volunteers comparing ToM and Phys conditions. For comparison the portion of area 8/9 which showed peak activation during ToM for normal controls is highlighted in red.

\section{Discussion}

The purpose of this study was to examine brain activity in relation to specific cognitive processes associated with theory of mind. We do not have a large enough sample to examine the more general abnormalities of brain structure or function found in autism in previous studies. ${ }^{6}$ Performance on the tasks used during scanning was at a similar level for the clinical and the normal control group. The exception was the poorer performance on ToM stories among patients with A sperger syndrome, a result which is expected from the theory of mind deficit hypothesis of autism. $N$ evertheless it should be noted that all A sperger subjects succeeded in correctly answering most of the test questions of the mentalizing stories and read these stories faster than the physical stories.

The comparison of story reading and unlinked sentences highlights areas involved in story integration and in the drawing of inferences. In our previous study ${ }^{10}$ the major difference between story and nonstory conditions involved the temporal poles bilaterally and the left superior temporal gyrus. These same areas were also activated in the A sperger volunteers, but for this group the difference between stories and sentences was significantly less pronounced in all regions. This suggests that subjects with A sperger syndrome process meaningful connected narrative and meaningless jumbled sentences in a more similar way than do controls.

Interestingly, our A sperger subjects were at least as good as previously scanned controls with unlinked sentences, but performed slightly worse on both story conditions. These findings fit a current theory suggesting that autism is characterized by a cognitive style of weak central coherence, a failure to integrate information in context to extract higher level meaning or gist. ${ }^{18}$ Piecemeal processing of stories, as of unlinked sentences, would be predicted by this account.

The most important comparison, between mentalizing (ToM) and non-mentalizing (Phys) stories, indi- cated a critical difference between the clinical and normal groups: the Asperger group did not show activation of the medial part of left prefrontal area 8/9 during ToM stories. A direct comparison of the groups (the group by condition interaction) confirmed that there was a significant difference in this region. These patients showed significant activation of a neighbouring area of left medial prefrontal cortex, Brodmann's area 9/10, when processing ToM stories, however. Controls also showed activation in this area, but to a far lesser extent than area 8/9. This partial overlap between the results from the two groups suggests that the A sperger subjects' mentalizing performance was subserved by a brain system in which one key component was missing.

$O$ ne explanation for this abnormal pattern of activation is that the $A$ sperger subjects were using a more general purpose reasoning mechanism in order to infer mental states. A rea 9, which covers a large expanse of cortex, has been implicated in a number of brain imaging studies of problem solving and general cognitive ability. ${ }^{19}$ The borderline portion of area $9 / 10$, activated in the present study, has not been linked to any specific cognitive function, however. $\mathrm{N}$ o macroscopic structural abnormalities of medial prefrontal cortex were present in the magnetic resonance imaging scans of the five A sperger volunteers, suggesting the possibility that the abnormal activation in this critical region resulted from structural damage in another part of an extended system or in microscopic abnormalities in intercellular connections. While frontal lobe dysfunction has been suggested for people with autism and Asperger syndrome, ${ }^{20,21}$ the present findings suggest the need for more specific task analysis of executive functions and a fractionation of the possible components and brain pathways involved.

\section{Conclusion}

This study has provided further evidence for a brain location which may be a key anatomical component of the 'theory of mind' system. It will be important to replicate this result with other appropriate subject groups. Further research will also be necessary to identify the other components of the system and the precise role that each plays. Since the brain areas identified so far are not uniquely human, it should also be possible to specify the precursors and components of 'theory of mind' abilities from studies of these brain regions in animals other than man.

ACKNOWLEDGEMENT: This work was carried out at the MRC Cyclotron Unit Hammersmith Hospital. We are extremely grateful for the use of these facilities. O.F., R.D., R.F. and C.F. are supported by the Wellcome Trust

\section{Received 23 August 1996;}

\section{accepted 25 September 1996}




\section{References}

1. Premack D and Woodruff G. Behav Brain Sci 4, 515-516 (1978).

2. Carruthers $P$ and Smith PK. Theories of Theories of Mind Cambridge: Cambridge University Press, 1996.

3. Baron-Cohen S, Leslie A and Frith U. Cognition 21, 37-46 (1985)

4. Baron-Cohen S, Leslie A and Frith U. Br J Dev Psychol 4, 113-125 (1986). 5. Gillberg C and Coleman M. The Biology of the Autistic Syndromes. London: MacKeith Press, 1992.

6. Bauman ML and Kemper TL, eds. The Neurobiology of Autism. Baltimore: J ohns Hopkins University Press, 1994.

7. Bailey A, Phillips W and Rutter M. J Child Psychol Psychiatry 37, 89-126 (1996)

8. Leslie A. Psychol Rev 94, 412-126 (1987).

8. Leslie A. Psychol Rev 94, 412-126 (1987).
9. Frith U, Morton J and Leslie A. Trends Neurosci 14, 433-438 (1991).

10. Fletcher $\mathrm{P}$, Happé F, Frith U et al. Cognition 57, 109-128 (1995).
11. Mazoyer BM, Tzourio N, Frak V et al. J Cogn Neurosci 5, 467-469 (1993). 12. Happé F. J Autism Dev Dis 24, 129-154 (1994).

13. Happé F. Child Dev 66, 843-855 (1995).

14. Frith $U$, ed. Autism and Asperger Syndrome. Cambridge: Cambridge University Press, 1991.

15. Frith U, Happé F and Siddons F. Soc Dev 3, 108-124 (1994).

16. Happé F. Cognition 48, 101-119 (1993).

17. Friston KJ , Worsley K, Frackowiak RSJ et al. Hum Brain Mapp 1, 214-220 (1994).

18. Frith U and Happé F. Cognition 50, 115-132 (1994).

19. Dolan RJ, Bench CJ, Brown RG et al. J Neurol Neurosurg Psychiat 55 768-773 (1992).

20. Ozonoff S, Pennington BF and Rogers SJ.J Child Psychol Psychiat 32 , 1081-1106 (1996).

21. Hughes C, Russell J and Robbins TW. Neuropsychologia 32, 477-492 Hughes
(1994). 